\title{
Consecuencias del tránsito de la escuela moderna a la posmodernidad: el nihilismo en estudiantes socialmente vulnerables
}

\author{
Consequences of the transition from modern school to \\ postmodernism: nihilism in socially vulnerable students
}

\section{*Sebastián Pereira Pinto}

Pereira, S. (2020). Consecuencias del tránsito de la escuela moderna a la posmodernidad: el nihilismo en estudiantes socialmente vulnerables. Revista Convergencia Educativa 7, julio, 1-14. DOI: http://doi.org/10.29035/rce.7.4

[Recibido: 04 mayo, 2020 / Aceptado: 18 mayo, 2020]

\section{RESUMEN}

En los últimos años hemos asistido al cambio de paradigma entre la modernidad y la posmodernidad. Esta última entrega a los seres humanos la promesa de una concepción del mundo mucho más amplia, donde las emociones y habilidades toman un rol principal. La educación academicista se encuentra en crisis, motivada por los cambios propuestos por la posmodernidad. Sin embargo, esta libertad de pensamiento se ha asentado sobre las bases de un mundo fuertemente desigual, hijo de la época moderna post revolución industrial. En este contexto, los estudiantes pertenecientes a grupos vulnerables en Chile han visto sus mentes invadidas por ideas de progreso y libertad del ser, pero han chocado contra la realidad brutal del sistema. En una sociedad fuertemente influenciada por la tecnología y el individualismo, es interesante dar notoriedad al fenómeno del nihilismo adolescente, el cual se aprecia fuertemente en contextos marginales segregados y olvidados. Además de reflexionar sobre esta problemática, se plantean algunas propuestas de solución a corto y largo plazo para evitar que este grupo de estudiantes siga siendo ignorado e invisibilizado.

Palabras clave: escuela, modernidad, posmodernidad, vulnerabilidad, nihilismo.

\footnotetext{
* Universidad del Bío Bío, Chillán, Chile.
} 


\begin{abstract}
In recent years, we have witnessed the change of paradigm between modernism and postmodernism. The latter delivers to humans the promise of a much broader worldview, where emotions and skills take a leading role. Academic education is in crisis, motivated by changes proposed by postmodernism. However, this freedom of thought has been based on a strongly unequal world, a child of the modern post-industrial revolution. In this context, students belonging to vulnerable groups in Chile have seen their minds invaded by ideas of progress and freedom of being, but have collided with the brutal reality of the system. In a society strongly influenced by technology and individualism, it is interesting to give visibility to the phenomenon of adolescent nihilism, which is strongly appreciated in marginal segregated and forgotten contexts. In addition to reflecting on this problem, some proposals for short and long-term solutions are put forward to prevent this group of students from being ignored and made invisible.
\end{abstract}

Key words: School, Modernism, Postmodernism, Vulnerability, Nihilism.

\title{
1. Introducción
}

La escuela clásica podría llamarse el logro más importante de la modernidad, entendiendo esta como ideas de racionalidad, concepciones estrictas y dogmáticas, y por sobre todo una mirada absolutamente academicista (Márquez, 2016). En este escenario predomina la lógica económica e intelectual, es decir, que lo único realmente importante de aprender es lo que pueda reportar algún beneficio material al sujeto o al sistema. Esta dualidad académico-económica es fruto de su contexto, la escuela moderna en la praxis solo requería formar obreros que tuvieran las capacidades de operar las nuevas tecnologías emanadas del período post revolución industrial (García-Lastra, 2013), además de reproducir y mantener los ideales nacionalistas de los nacientes estados modernos.

Este modelo de escuela funcionó durante siglos, amparado por su efecto colateral, adoctrinar a las masas, permitiendo moldearlas a voluntad y creando las condiciones necesarias para el auge del sistema neoliberal actual. Esto acrecentó aún más las desigualdades existentes, creando una falsa idea de progreso en base a la educación. Si agregamos la creciente marginalidad social, tenemos un grupo altamente vulnerable adoctrinado para ser mano de obra barata y obediente gracias a la coacción de la escuela moderna.

Paralelamente, a mediados del siglo XX se desarrolló una revolución del pensamiento, fruto de la caída de las grandes ideologías y por sobre todo, el avance de las tecnologías de la información, las cuales democratizaron el acceso a la ideas como nunca en la historia. Ante la crisis de los dogmas e ideologías entra en escena el concepto de posmodernidad, el cual viene a cuestionar los cimientos de la escuela moderna, gracias a su visión del mundo muchísimo más abierta, que reconoce la multiculturalidad, la diferencia, el libre pensamiento, promueve la creatividad, la solidaridad, etc. (Moret, 2016). Esta libertad de pensamiento 
Pereira, S. (2020). Consecuencias del tránsito de la escuela moderna a la posmodernidad: el nihilismo en estudiantes socialmente vulnerables. Revista Convergencia Educativa. 7, julio, 1-14. DOI: http://doi.org/10.29035/rce.7.4

trae consigo el cuestionamiento de todo lo establecido, mientras exista algo o alguien que apoye determinada idea, es posible hacer esta como propia.

Sin embargo, y pese a esta revolución del pensamiento, la lógica materialista instaurada por el capitalismo se ha profundizado con el tiempo. Hoy más que nunca el mercado determina qué conocimiento es útil frente a sus necesidades prácticas, solo con la diferencia que el actual neoliberalismo deja de lado el reforzamiento nacionalista en pos de privilegiar la capacitación adecuada de sus engranajes para la producción de riqueza. Hoy tenemos el choque irremediable de concepciones completamente posmodernas contra un sistema moderno en su base, el cual ha creado una situación de confusión sin precedentes en los educandos en proceso de formación. Dicha contradicción es mucho más visible en los grupos más vulnerables de la sociedad, quienes crecen con la promesa neoliberal de mejora y crecimiento, pero finalmente solo logran experimentar el desencanto y desaliento propios de una sociedad que les ofrece todas las oportunidades, pero no está dispuesta a ceder ninguna.

\section{De una escuela moderna a una posmoderna}

La posmodernidad es un proceso cultural iniciado en la década de los setenta que involucra las artes visuales, la música, la tecnología, la ciencia y la filosofía (Mello, 2016), reformula las ideas modernistas de comprensión bajo leyes absolutas, de correlaciones absolutamente causales, para dar paso a una autonomía de pensamiento que no necesariamente separa las cosas entre causa y efecto. El posmodernismo podría conceptualizarse como ecléctico en el sentido que no acepta un amarre absoluto ante ningún credo, ideología o estructura predeterminada (Mello, 2016). Algunos autores como Moret (2016) no asignan una fecha cronológica a este cambio, sino más bien dan una impresión más utilitaria del cambio de pensamiento:

Cuando los avances tecnológicos, intelectuales, económicos, políticos y sociales dejaron de llevar al Hombre hacia una situación cada vez mejor que la anterior, es decir, cuando la idea de Progreso dejó de ser eficiente, es cuándo la Modernidad se acabó, es cuando la Postmodernidad se inició (Moret, 2016, p.341).

Como una fuerza que penetra todas las esferas de la sociedad, nos encontramos ante una revolución de las ideas en toda la norma, dejar atrás el pasado positivista y racional para abrazar paulatinamente ideas muchísimo más libertarias, sobre todo en el ámbito de la libertad de pensamiento y decisión. La razón absoluta sobre las cosas da paso a un escepticismo crónico frente a las ideas que deambulan en la mente de las personas (Moret, 2016). Este quiebre nos lleva a dudar si las ideas de antaño nos llevaran al progreso en este nuevo mundo difuso y altamente cambiante. 
Pereira, S. (2020). Consecuencias del tránsito de la escuela moderna a la posmodernidad: el nihilismo en estudiantes socialmente vulnerables. Revista Convergencia Educativa. 7, julio, 1-14. DOI: http://doi.org/10.29035/rce.7.4

Según Lipovetsky (2011) nos encontramos ante una cultura planetaria, una concepción ecuménica, pero sin dejar de lado lo local, algunas características de esta inclinación:

La revolución de las tecnologías de la información y comunicación (TIC); La organización de vastas redes mediáticas transnacionales; La ampliación de industrias y entidades culturales canalizadas hacia un creciente mercado globalizado; La vivencia local de las aquellas: la parte de la cultura globalizada no pone fin a los sentimientos nacionales ni a la territorialidad urbana (Cit. en Daros, 2017, p.15)

Podemos inferir que la posmodernidad rescata tanto lo local como lo global, pero hace consiente al sujeto que es parte de un todo, una expresión superior de su propia existencia, en cierto sentido, ocurre un fenómeno de despersonalización. La realidad deja de ser una estructura fija y pasa a ser un fenómeno dinámico, en parte gracias a las nuevas tecnologías y la ampliación sin límites de la información a disposición.

El posmodernismo en educación puede expresarse como un rechazo a la escuela clásica portadora de ideales morales y éticos absolutos. Este flujo de nuevas ideas se manifiesta en la pérdida de confianza en la institución, la escuela deja de ser un referente de civilidad, como lo era en el pasado (Mosquera y Ramírez, 2020).

Este cambio es propiciado por una intensificación del ideal de ciudadano como un consumidor, engranaje vital para el sistema neoliberal. Como bien plantea Lipovetsky (2011) la escuela ya no es la encargada de transmitir valores, sino más bien reproducir las necesidades del contexto en que se inserta:

La escuela ya no tiene por misión superior inculcar valores morales, republicanos y patrióticos: funciona como un servicio que se ofrece a consumidores exigentes y críticos que deciden entre escuela privada y escuela pública. Las humanidades ya no atraen a las élites: las grandes escuelas de comercio han tomado el relevo (Cit. en Daros, 2017, p.15)

El capitalismo globalizado ya no es un fenómeno cultural fundado en ideologías, sino creador de un relato de progreso en base a la igualdad de oportunidades ficticia, puesto que oculta las grandes desigualdades que aquejan a una sociedad dominada por las elites económicas (Daros, 2017).

Sin embargo, esto no debiera suponer el fin de la escuela, sino una reformulación total siguiendo las ideas de diversidad, entendimiento e inclusividad del posmodernismo (González, 2014). El camino más sensato sería que las posturas modernas y posmodernas lograran un diálogo, donde no se pierdan los beneficios que ambas pueden entregar al proceso de enseñanza-aprendizaje, un puente de un sistema a otro eliminaría las dificultades de un cambio súbito de paradigma (Mosquera y Ramírez, 2020). 
Pereira, S. (2020). Consecuencias del tránsito de la escuela moderna a la posmodernidad: el nihilismo en estudiantes socialmente vulnerables. Revista Convergencia Educativa. 7, julio, 1-14. DOI: http://doi.org/10.29035/rce.7.4

La nueva forma de entender la sociedad, como un acceso inmediato a la gratificación vacía, falta de tolerancia a la frustración, etc., hace incongruente al adolescente posmoderno adecuarse a las lógicas disciplinares de la escuela moderna, donde el resultado es todo, sin tener en cuenta individualidades o capacidades distintas (Segni, 2018). Además de tener en cuenta habilidades no reconocidas en la modernidad, la educación posmoderna plantea una instrucción en constante dinamismo, como bien plantea García-Lastra (2013):

Una de las ideas fundamentales que debe tenerse en cuenta a la hora de abordar las características de la escuela del siglo XXI es el carácter efímero de los conocimientos por transmitir y la consecuencia de este hecho sobre los procesos de enseñanza-aprendizaje que en ella tienen lugar. Sin negar la importancia de la transmisión de las bases de una alfabetización básica y de contenidos fundamentales para lograr la pertenencia a una cultura común, se debe considerar que muchos de los conocimientos válidos en la actualidad habrán caducado en poco tiempo (García-Lastra, 2013, p.203).

\section{El fenómeno colateral de la posmodernidad: Nihilismo adolescente}

Como las ideas no son constructos inamovibles y eternos, menos en esta era de la individualidad, cada sujeto tiene la libertad de moverse entre las corrientes que desee. Esta sensación, además de provocar una sensación de libertad cognitiva, trae consigo un subproductos no deseados, como la apatía, el desapego, el desinterés, el nihilismo, si quisiéramos apoyarnos en las ideas de Nietzsche y Heidegger (Vattimo, 2015).

Este nihilismo posmoderno penetra a todas las generaciones, tanto adultos, adolescentes y niños, etc. Es necesario enfrentar este cambio paradigmático desde los centros formadores, puesto que esta postura filosófica en edades adultas puede ayudar a deconstruir el pensamiento y provocar un cambio positivo, sin embargo, este nihilismo es preocupante en los adolescentes actuales, sobre todo en capas más vulnerables de la sociedad.

Determinar los cánones que definen estos contextos es complejo, pero es posible aportar con una definición acotada de lo que es la vulnerabilidad social:

Un proceso al cual puede concurrir cualquier persona, grupo o comunidad que en un momento determinado se encuentre en una situación desfavorecida o de desventaja con respecto a otras personas, grupos o comunidades; y que las causas, consecuencias y efectos de dicha desventaja se investigan teniendo en cuenta dos elementos; los riesgos que se enfrentan; y los activos con los que se cuenta para mitigar el impacto de los mismos, cuyas características y puesta en funcionamiento marcarán diferentes niveles de vulnerabilidad ante los riesgos [...] En este sentido, el riesgo social se analiza a partir de tres componentes que va a definir la vulnerabilidad 
Pereira, S. (2020). Consecuencias del tránsito de la escuela moderna a la posmodernidad: el nihilismo en estudiantes socialmente vulnerables. Revista Convergencia Educativa. 7, julio, 1-14. DOI: http://doi.org/10.29035/rce.7.4

social: la peligrosidad o amenaza social, la exposición y los activos. La peligrosidad o amenaza social se entiende como el conjunto de aspectos sociales de un fenómeno que inciden en el riesgo y que se concreta por la severidad del fenómeno y su probabilidad de ocurrencia; la exposición está referida a las personas, grupos o comunidades localizadas en un ámbito territorial y/o nivel social y económico próximas a un peligro social; y los activos son los elementos con los que cuenta una persona, grupo y comunidad para enfrentar y/o mitigar los riesgos y/o sus consecuencias. (Cit. en Ferrada et al., 2015, p. 30-31)

Además de lo anteriormente expuesto, vulnerabilidad se podría definir como un escaso capital cultural y altas dosis de marginalidad, donde la sobrevivencia es del día a día, donde hasta la esperanza ha escapado, al sujeto lo invade la pérdida de razón para existir. A esto se suma la marginación de sus pares "no vulnerables" los cuales no quieren compartir el mismo espacio educativo y migran hacia lugares con una mejor oferta educativa, produciendo una segregación orgánica entre estudiantes. Esto no ocurre por la infraestructura o falta de recursos, o por inexperiencia del personal, sino por el roce que en teoría van a tener con mejores componentes sociales (Bellei, 2013). Este fenómeno aumenta aún más la sensación de marginación, ya que pese a noción existente de igualdad de oportunidades, estas en la práctica no son visibles de ninguna forma.

Si bien las ideas de apatía son generales a la población posmoderna, el sujeto que vive una realidad más acomodada, puede tener al menos la intención de perpetuar dichos privilegios. Por ende aunque no tenga entusiasmo persé, puede al menos encontrar en perpetuar el status quo de su clase una motivación para evitar la apatía. Caso contrario con los adolescentes de clases vulnerables, que solo han conocido la miseria durante todas sus vidas y en estricto rigor no vislumbran un escenario distinto en un futuro próximo. Si en el modelo modernista la reproducción de la clase obrera era el fin, en el posmodernismo se le entrega a las nuevas generaciones la potestad de elegir no sentir conexión o apego con cosa alguna, es decir, una libertad de pensamiento mal entendida.

A nivel concreto, es posible apreciar que la tasa de deserción escolar se ha mantenido o disminuido en Chile, sin embargo, en sectores más vulnerables está ha aumentado, sobre todo por razones anexas a lo económico (CASEN, 2017). Además de los múltiples factores socioeconómicos, esto puede relacionarse al componente motivacional que experimentan los jóvenes de este estrato. Actualmente no es posible atribuir la falta de interés o fracaso escolar solamente a las habilidades propias del alumno, sino a variables cognitivas y motivacionales (Sáez, 2013). La apatía o desinterés de los estudiantes nace de la imposibilidad de ver sus metas cumplirse. La sensación de vivir en un mundo donde hagas lo que hagas no se cumplen los anhelos y metas, deriva irremediablemente en una carencia de sentido frente a cualquier actividad (Aguilar et al., 2015). 
Pereira, S. (2020). Consecuencias del tránsito de la escuela moderna a la posmodernidad: el nihilismo en estudiantes socialmente vulnerables. Revista Convergencia Educativa. 7, julio, 1-14. DOI: http://doi.org/10.29035/rce.7.4

Una de las ideas más difundidas del ideal posmodernista es la llamada meritocracia, la cual difunde la idea de que el éxito no está basado en los lazos sanguíneos o jerárquicos, sino que en el mérito personal que cada sujeto puede tener. Esta idea de meritocracia está muy difundida en los adolescentes (Ochoa y Orbeta, 2017), sin embargo, en los últimos años esta idea ha caído en critica constante, debido a la liberalización del pensamiento, donde las personas se han dado cuenta que pese al esfuerzo personal esta idea de éxito no es real. La nueva forma de ver el mundo ha transformado para mal las ideas preconcebidas de "triunfar". Si ni siquiera los lazos sanguíneos, ni la meritocracia son garantías de éxito, es una tónica caer en la desesperanza temprana respecto a la vida.

Es sabido que el aprendizaje no está reducido únicamente a la dimensión cognitiva, intenciones, metas, motivos y creencias tienen un efecto directo en como los estudiantes enfrentan la tarea de educarse. Existe una correlación directa entre lo cognitivo y lo motivacional, uno sin el otro desencadena irremediablemente el fracaso de la aprehensión de determinado objetivo de aprendizaje (Sáez, 2013).

Este fenómeno tiene muchas aristas e investigaciones por visualizar, ya que escapa al dominio exclusivo de la educación. Esta apatía no es solo por el acto de aprender en la escuela, sino que atañe a cualquier ámbito en la vida de los jóvenes posmodernos. Pese a lo difícil que puede resultar rescatar una mente de esta apatía crónica, es menester de este estudio proponer algunas soluciones, que, si bien no vienen acabar con el problema completamente, si pueden ser un aporte práctico para enfrentar el desafío constante de encantar a esta nueva generación de educandos. Esta misión no es solo para mantener o adecuar las dinámicas escolares, sino para entregar herramientas concisas a los estudiantes en su diario vivir, puesto que este nihilismo puede resultar profundamente peligroso para la evolución de las sociedades en el futuro.

\section{Propuestas de intervención frente a la problemática}

Encontrar soluciones a un problema de motivación filosófica como la razón de vivir es bastante complejo. Si bien los jóvenes quizás no están al tanto de las implicancias filosóficas de su sentir, es claramente una problemática enraizada en este campo de las ciencias sociales. Teniendo en cuenta lo complejo de un desgano general frente a la vida, sin querer dar de ninguna forma una solución absoluta y final, es posible proponer algunas alternativas prácticas para enfrentar a estos jóvenes posmodernos y quizás lograr que puedan reencantarse con la educación y con la vida.

Una característica propia de esta generación es que dejaron de ser hace mucho tiempo análogas, la digitalización de la sociedad es una realidad hace ya varios años. Para los niños y adolescentes que estudian actualmente en las escuelas formales la tecnología es su idioma natal (Flores et al., 2013). Teléfonos inteligentes, redes inalámbricas, tablets, laptops, etc., son parte del lenguaje inicial de cualquier joven actual. Es numerosa la literatura que demuestra la eficacia de la aplicación de TIC en el proceso enseñanza- 
Pereira, S. (2020). Consecuencias del tránsito de la escuela moderna a la posmodernidad: el nihilismo en estudiantes socialmente vulnerables. Revista Convergencia Educativa. 7, julio, 1-14. DOI: http://doi.org/10.29035/rce.7.4

aprendizaje, en los más diversos contextos (Toribio, 2019, Hernández, 2017, Cuban, 2016). Por esta razón es necesario adecuar el discurso de un profesor análogo a un estudiante digital. Pese a lo que se pueda pensar, el acceso a la tecnología es bastante democrático, alumnos de sectores vulnerables si cuentan con tecnología necesaria para llevar a cabo una adecuada clase con TIC, solo haría falta una reeducación de estos, ya que su manejo solo estaría limitado a redes sociales e información básica (Granado, 2019).

Es imprescindible aprovechar esta oportunidad, ya que a nivel fisiológico está demostrado que el cerebro procesa mucho mejor la información cuando la recibe no solo del canal visual, sino también del auditivo y demás sentidos (Flores et al., 2013). Por lo mismo, integrar TIC como Socrative, Google Docs, YouTube, o inclusive Realidad aumentada (RA) podría ser una estrategia de enorme valor didáctico. La RA además de favorecer el aprendizaje por descubrimiento, entregar información y/o visitar lugares históricos o fantásticos, permite la interacción con cosas que difícilmente podrían estar al alcance de los estudiantes (Lorenzo \& Scagliarini, 2018). Acá la tarea es poder capacitar al docente en el manejo de tecnologías de la información innovadoras y atrayentes, un desafío permanente de actualización para lograr encantar a estos jóvenes.

La figura del docente en todo este proceso es vital, por eso es bastante interesante rescatar el concepto de saberes docentes, que se podrían definir como el conjunto de saberes que tenga un docente, como el disciplinar, las competencias en el manejo de grupo, valores, habilidades, actitudes, etc., es decir, cualquier ámbito del campo de la docencia, donde el docente aplique conocimientos aprehendidos tanto en su formación como en su práctica docente (Tardif, 2010). De los saberes descritos por Tardif el más apropiado sería el saber pedagógico, el cual tiene directa relación las herramientas que debe poseer un docente al enfrentarse a un contexto particularmente complicado y estudiantes claramente sin el mayor interés en aprender, ya que focaliza la acción en las herramientas que tiene un docente en relación a la propia experiencia de un contexto especifico (Tardif, 2010). Según Merellano-Navarro et al. (2019) la construcción del saber pedagógico es un proceso individual y a la vez colectivo. Comienza con la formación inicial docente, de forma particular, para luego continuar construyéndose dinámicamente durante el transcurso de toda su docencia, en una relación dialéctica entre teórica y práctica. Este saber requiere necesariamente de un profesional reflexivo, capaz de comprender las implicancias propias del conocimiento que va adquiriendo. El saber pedagógico implica el ejercicio de una práctica reflexiva, comprometida, con sentido e intencionalidad (Merrellano-Navarro et al., 2019). Finalmente, el autor concluye que este ejercicio sistemático, permite al docente generar conocimiento propio desde su propia práctica pedagógica, con el objeto de poder transformarla. La razón de usar este concepto es que un correcto estudio de estos saberes, puede detectar las deficiencias de los docentes actuales e intentar "equilibrar la balanza" entre las debilidades y las fortalezas que tengan los profesores. Entender este cúmulo de conocimientos es 
Pereira, S. (2020). Consecuencias del tránsito de la escuela moderna a la posmodernidad: el nihilismo en estudiantes socialmente vulnerables. Revista Convergencia Educativa. 7, julio, 1-14. DOI: http://doi.org/10.29035/rce.7.4

posmodernista, puesto que asume al profesor como un sujeto dinámico (Tardif, 2010) que nunca deja de aprender ni mejorar su experiencia docente.

Otra variable importante de ser analizada y remediada es el auto concepto que poseen los estudiantes sumidos en la pobreza y/o marginalidad. El nivel o estatus socioeconómico incide directamente en el auto concepto juvenil. El saberse miembro de un determinado grupo social vulnerable incide directamente en las representaciones que dicho grupo interioriza (Antonio-Agirre et al., 2019). El auto concepto es una de las variables más importantes dentro del ámbito de la personalidad, la propia concepción delimita la capacidad que tiene el sujeto a tener un aprendizaje mucho más eficaz y duradero (Sáez, 2013). Mientras mejor auto concepto tenga el estudiante, es decir, una mayor apreciación de sí mismo, metas claras y/o confianza en su propia superación, mayor será el avance. Es necesario eliminar de la ecuación al ambiente lo más posible, intentar que el estudiante se haga cargo de su propio destino. Que más allá de la realidad contextual, saber que la superación si puede ser una herramienta clave de éxito o realización personal. Estudios como el de Sáez (2013) demuestran que, a mayor auto concepto, mayor es la calidad y consecuencia en sus vidas gracias al aprendizaje.

El aspecto motivacional es uno de los principales desafíos de la educación posmoderna debido a que los estudiantes actuales son altamente individualistas y cuidadores de su propia individualidad. Es necesario encontrar las alternativas necesarias para captar la atención de todos ellos, o sea, educar la diversidad. En Chile, se ha trabajado esta problemática, pero las soluciones son aún complejas y distantes. Propuestas como la de Hernández \& Lino (2017), donde se ofrece una completa estructura funcional de propuestas de motivación que están aún lejos de aplicarse masivamente en estudiantes vulnerables. Los mismos autores explican que hay variada literatura frente al tema, pero que sería necesario llevar estas a un plano más empírico.

Las diferentes propuestas aquí planteadas deben ser aplicadas de manera conjunta. La investigación en este sentido debe apuntar a detectar los fenómenos específicos de cada contexto, para así elaborar la estrategia más atingente al tipo de estudiante. Sin duda el docente sigue teniendo un papel principal en esta nueva era, sin embargo, esta vez la propuesta es evitar la verticalidad de la relación, estableciendo un vínculo de confianza, empatía y solidaridad con los estudiantes. Como sociedad debemos hacernos cargo de este sentir, puesto que el desapego, la apatía y el desencanto son un enemigo silencioso que puede desencadenar una serie de productos no deseados en nuestra sociedad. Lo que originalmente estaba pensado para liberarnos de la estructura moderna, podría desencadenar la perdida de sentido total para esta generación y las que vendrán. 
Pereira, S. (2020). Consecuencias del tránsito de la escuela moderna a la posmodernidad: el nihilismo en estudiantes socialmente vulnerables. Revista Convergencia Educativa. 7, julio, 1-14. DOI: http://doi.org/10.29035/rce.7.4

\section{Conclusiones}

La posmodernidad ha transformado al modelo de escuela clásica en algo obsoleto (González, 2014), sin embargo, llama la atención que este fenómeno no es el culpable directo de los sentimientos nihilistas en las nuevas generaciones, sino más bien la mercantilización de la educación, el sistema neoliberal transformando los viejos ideales de crecimiento intelectual en títulos y competencias transables, una reafirmación vacía del yo frente a los demás (González, 2017).

Por una parte, tenemos un sistema educativo que al igual que en su origen, intenta perpetuar las estructuras básicas del contexto que le toca vivir (Carter-Thuillier \& Moreno, 2017) y por otra, está la idea de progreso material, incompatible con la realidad segregadora del mismo. Bellei (2013) correlaciona directamente los efectos de la segregación socioeconómica con las lógicas de mercado imperantes producto del sistema neoliberal, $\mathrm{y}$, por consiguiente, todos los vicios que pueda traer consigo esta estructura educativa.

Este modelo, vive en una constante tensión entre quienes defienden las políticas de mercado y los sectores sociales que se resisten a ellas (González, 2017). Este segundo grupo se ve agobiado por la lógica de "ser empresario de uno mismo" (Hidalgo et al., 2017) donde uno es responsable de su propio éxito, sin tomar en consideración el componente colaborativo, primordial para enfrentar los desafíos de nuestra época.

Los jóvenes de hoy viven bajo la promesa de lograr metas en teoría alcanzables, impulsadas por la capacidad de acción y elección, pero perdidos sin una guía adecuada de estas posibilidades (Hidalgo et al., 2017). La socialización y la importancia del trabajo en equipo emergen como vitales a la hora de hacer frente al individualismo crónico de la sociedad neoliberal (Carter-Thuillier \& Moreno, 2017). Esta sería una forma de evitar la despreocupación frente al futuro propio en un sistema apático que solo hace apología del "yo". (Hidalgo et al., 2017).

Ante este escenario es fundamental el desarrollo de la crítica en todos los actores de la comunidad educativa (Ochoa y Orbeta, 2017) ya que es la única forma de comprender la complejidad del tejido social. Comprender que el individualismo apático no es un camino valido, sino más bien una respuesta desesperanzada a la promesa neoliberal (Hidalgo et al., 2017).

Ante este escenario sistémico complejo, la colaboración, solidaridad, reflexión y empatía parecieran ser los caminos más adecuados para alejarnos del afán individualista y comportarnos como lo que somos, una sociedad que ha demostrado que el trabajo conjunto es la mejor receta para que el desarrollo humano se enfoque en el bienestar general. 
Sería muy interesante que este trabajo fuera tomado como punto de partida para futuras investigaciones empíricas, donde pueda constatarse a nivel local de cada establecimiento la realidad de los estudiantes en relación a su propio contexto, ya que ningún contexto es igual al otro, por más que compartan condiciones similares.

La posmodernidad ha sido aliada del neoliberalismo en los últimos años, pero quizás sus herramientas de cooperación y reflexión serán la llave para que en un futuro nuestros estudiantes puedan configurar sus destinos a voluntad, con una mirada colaborativa y proactiva de sus propias vidas.

\section{REFERENCIAS BIBLIOGRÁFICAS}

Aguilar, Y., Valdez, J., González, N., Rivera, S., Carrasco, C., Gómora, A., Pérez, A., \& Vidal, S. (2015). Apatía, desmotivación, desinterés, desgano y falta de participación en adolescentes mexicanos. Enseñanza e Investigación en Psicología, 20(3), 326-336. Recuperado de https://www.redalyc.org/pdf/292/29242800010.pdf

Antonio-Agirre, I., Axpe, I., \& Septien, A. (2019). La influencia del estatus socioeconómico y cultural en la relación entre el auto concepto y la inteligencia emocional percibida en la adolescencia. European Journal of Education and Psychology, 13(1), 235-250. DOI: https://doi.org/10.30552/ejep.v13i1.291

Bellei, C. (2013). El estudio de la segregación socioeconómica y académica de la educación chilena. Estudios pedagógicos (Valdivia), 39(1), 325-345. DOI: http://dx.doi.org/10.4067/S071807052013000100019

Carter-Thuillier, B., \& Moreno, A. (2017). Globalización económica, postmodernidad y sistema educativo: contradicciones y alternativas desde una Educación Física crítica. Estudios pedagógicos (Valdivia), 43(3), 103-117. DOI: https://dx.doi.org/10.4067/S0718-07052017000300006

CASEN (2017). Observatorio Social. Previsión social: sintesis de resultados. Santiago de Chile: Ministerio de Desarrollo Social y Familia. Santiago, Chile. http://observatorio.ministeriodesarrollosocial.gob.cl/casen-multidimensional/casen/casen 2017.php

Cuban, L. (2016). Problemas recurrentes a los que deben enfrentarse los investigadores cuando estudian la adopción y el uso de las TIC en el aula. En J. Mominó y C. Sigalés, El impacto de las TIC en la educación: Más allá de las promesas (pp. 27-41). Barcelona: Editorial UOC. Recuperado de http://reader.digitalbooks.pro/book/preview/43319/x03_ImpactoTIC_cap01 
Pereira, S. (2020). Consecuencias del tránsito de la escuela moderna a la posmodernidad: el nihilismo en estudiantes socialmente vulnerables. Revista Convergencia Educativa. 7, julio, 1-14. DOI: http://doi.org/10.29035/rce.7.4

Daros, W. (2017). Posmodernidad y educación en la concepción de G. Lipovetsky. Revista De Filosofía De La Universidad De Costa Rica, 56(144), 11-20. Recuperado de https://revistas.ucr.ac.cr/index.php/filosofia/article/view/28326

Ferrada, D., Villena, A., \& Turra, O. (2015). Transformar la formación: las voces del profesorado. Santiago: RIL Editores.

Flores, I., González, G., \& Rodríguez, I. (2013). Estrategias de enseñanza para abatir la apatía del alumno de secundaria. Revista iberoamericana para la investigación y el desarrollo educativo, 10. Recuperado de http://1-11.ride.org.mx/index.php/RIDESECUNDARIO/article/view/316

García-Lastra, M. (2013). Educar en la sociedad contemporánea, hacia un nuevo escenario educativo. Convergencia. Revista De Ciencias Sociales, 62, 199-220. Recuperado de https://www.redalyc.org/pdf/105/10525851011.pdf

González, J. F. (2014). El anacronismo de la escuela según la posmodernidad. CISMA, Revista del Centro Telúrico de Investigaciones Teóricas, 5, 1-23. DOI: http://dx.doi.org/10.2139/ssrn.2768472

González, N. (2017). La lucha simbólica por la educación en la globalización neoliberal. Revista Brasileira de Educação, 22(71), e227171. DOI: https://doi.org/10.1590/s1413-24782017227171

Granado Palma, M. (2019). Educación y exclusión digital: los falsos nativos digitales. Revista De Estudios Socioeducativos, ReSed, (7), 27-41. Recuperado de https://revistas.uca.es/index.php/ReSed/article/view/4404

Hernández, E., \& Lino, E. (2017). La motivación y su incidencia en el rendimiento escolar (tesis de licenciatura). Universidad de Guayaquil, Ecuador. Recuperado a partir de http://repositorio.ug.edu.ec/handle/redug/24904

Hernández, R. (2017). Impacto de las TIC en la educación: Retos y Perspectivas. Propósitos y Representaciones, 5(1), 325-347. DOI: http:// dx.doi.org/10.20511/pyr2017.v5n1.149

Hidalgo, B., Dueñas, E., \& Molina, G. (2017). Una aproximación sociológica a la depresión, Flexibilidad, hiperindividualización y medicalización en la sociedad posmoderna. Revista sociológica de pensamiento crítico, 11(2), 9-33. Recuperado de http://www.intersticios.es/article/view/17702

Lorenzo, G., \& Scagliarini, C. (2018). Revisión bibliométrica sobre la realidad aumentada en Educación. Revista general de información y documentación, 28(1), 45-60. DOI: https://doi.org/10.5209/RGID.60805

Márquez Carrillo, J. (2016). La escuela moderna y los orígenes de la formación cívica y moral en México, 1790-1835. Letras históricas, (15), 43-71. DOI: http://dx.doi.org/10.31836/lh.15.6095 
Pereira, S. (2020). Consecuencias del tránsito de la escuela moderna a la posmodernidad: el nihilismo en estudiantes socialmente vulnerables. Revista Convergencia Educativa. 7, julio, 1-14. DOI: http://doi.org/10.29035/rce.7.4

Mello, G. (2016). Pos-Modernismo: entre a Crítica e a Ideología. Trans/Form/Ação, 39(1), 233-258. DOI: https://dx.doi.org/10.1590/s0101-317320160001000011

Merellano-Navarro, E., Almonacid-Fierro, A., y Oyarce, M. (2019). Resignificando el saber pedagógico: una mirada desde la práctica docente. Educação e Pesquisa, 45. DOI: http://dx.doi.org/10.1590/s16784634201945192146

Moret, R. (2016). La posmodernidad: intento de aproximación desde la historia del pensamiento. Bajo Palabra. Revista de filosofía, 2(7), 339-348. Recuperado de https://revistas.uam.es/bajopalabra/article/view/3243

Mosquera, C., \& Ramírez, J. (2020). El desencantamiento de la Escuela: sus cambios y zarandeos en la era posindustrial. Revista Interamericana de Investigación, Educación y Pedagogía, 13(1), 45-61. DOI: https://doi.org/10.15332/25005421

Ochoa, M., \& Orbeta, C. (2017). Discursos sobre clase social y meritocracia de escolares vulnerables en Chile. Cadernos de Pesquisa, 47(164), 496-518. DOI: https://dx.doi.org/10.1590/198053143752

Sáez, F. (2013). Mecanismos cognitivos motivacionales asociados a la autorregulación del aprendizaje. PAIDEIA, 53, 59-72. Recuperado de https://www.researchgate.net/publication/318851063_MECANISMOS_COGNITIVOS_MOTIVACIONALES_ASOCIADOS_A_LA_AUTORREGULACION_DEL_APRENDIZAJE_COGNITIVE_MOTIVATIONAL_MECHANISMS_ASSOCIATED_WITH_SELF-REGULATED_LEARNING

Segni, S. (2018). Releyendo Adolescencia, posmodernidad y escuela: Veinte años después. Revista Observatório, 4(2), 480-499. DOI: https://doi.org/10.20873/uft.2447-4266.2018v4n2p480

Tardif, M. (2010). Los saberes del docente y su desarrollo profesional. Madrid: Narcea.

Toribio, M. (2019). Importancia del uso de las TIC en educación primaria. Atlante: Cuadernos de Educación y Desarrollo. Recuperado de https://www.eumed.net/rev/atlante/2019/02/uso-tic-primaria.html

Vattimo, G. (2015). El fin de la modernidad. Nihilismo y hermenéutica en la cultura posmoderna. Barcelona: Gedisa. 
Pereira, S. (2020). Consecuencias del tránsito de la escuela moderna a la posmodernidad: el nihilismo en estudiantes socialmente vulnerables. Revista Convergencia Educativa. 7, julio, 1-14. DOI: http://doi.org/10.29035/rce.7.4

\section{Datos de correspondencia}

Sebastián Pereira Pinto

Magíster en Educación (c)

Universidad del Bío Bío

Chillán, Chile

Avda. Brasil 1180,

Región de Ñuble, Chile

ORCID: https://orcid.org/0000-0002-5145-020X

Email: sepereirapinto@gmail.com

(C) (†) (-) Esta obra está bajo una Licencia de Creative Commons Reconocimiento-Nocomercial- 